\title{
Research on Brain-Computer Interface System Based on DSP and Zigbee
}

\author{
Yong Sun ${ }^{1+}$, Xiaoqin $\mathrm{Li}^{1}$, Guoming Lin ${ }^{1}$, Jinlong Zheng ${ }^{1}$, Hongxia Hou ${ }^{1}$ \\ ${ }^{1}$ Tianjin Key Laboratory of Information Sensing \& Intelligent Control, Tianjin University of Technology \\ and Education, Tianjin300222, China.
}

\begin{abstract}
The brain-computer interface (BCI) system creates a new communication channel between the computer and the brain, which can be used to exchange information between the human brain and the external environment or control peripheral equipments. And it is dependent on computers or other electronic devices instead of the peripheral nerves and muscle tissue. This topic carries on the research of BCI system based on DSP and Zigbee. Through the acquisition of the human's steady state visual evoked potential (SSVEP), which will be translated into control commands and sent to household appliances (such as electric fans, curtains, lamps, etc.) via the wireless two-way communication module of Zigbee to make the control of the furniture through the brain come true. The experiment reveals that the recognition rate of the designed $\mathrm{BCI}$ system can reach more than $85 \%$, and the consuming time of sending a single control command is less than $5 \mathrm{~s}$. It is proved that the scheme of this system is feasible and has high application value.
\end{abstract}

Keywords: BCI, SSVEP, DSP, Zigbee.

\section{Introduction}

The brain computer interface (BCI) is a non-muscle communication system that allows his/her brain to communicate directly with the environment [1]. Connecting the external environment and the brain, it employs computers or other electronic devices to establish a new way of information exchange and control, that without the help of peripheral nerves and muscle tissue. It provides a new approach of communication with the outside world for patients who have no full muscle control functions as well. A complete BCI system consists of four parts: signal acquisition, signal feature extraction, signal classification and external control equipment. The acquisition accuracy, speed and stability of the BCI system are the main problems [2].

There are two issues that must be considered when connecting the brain with external devices. The first one is to choose appropriate sensors(electrodes) to effectively detect electroencephalogram(EEG) that reflects human's intentions. Considering the practicality and the speed of the sensor comprehensively, almost all BCI systems use sensors on the scalp(on the surface of the brain) to detect electrophysiological signals. There are also some researches use the signal collected from the cerebral cortex. For humans, most studies of EEG recordings are limited to the scalp in terms of safety and stability. Another one is the definition of communication language (ie, EEG characteristics in time domain or frequency domain measured at a specific location). Then, just like other communication systems, users can communicate using this language symbol after detecting these symbols by computers [3-4].

\section{Design of the BCI System Based on DSP}

A BCI system based on DSP has established a portable system adopted steady-state visual evoked potentials (SSVEP) with high precision, high speed and high real-time performance. Compared with the

\footnotetext{
Corresponding author. Tel.: + 13752295669.

E-mail address: sunyong10@126.com.
} 
existing brain-computer interface system based on PC, this system has the advantages of small size, portability and practicality.

The designed system is mainly composed of a visual stimulator module, an EEG recognition module and a control module. As is shown in Fig. 1, it is the system block diagram.

Visual stimulator module: This part is an external device that provides visual stimuli, using LED as a stimulator to generate accurate stimulation frequency. The subject stares at the stimulator with different frequencies to induce the SSVEP. Visual stimulators in this system generate frequencies of $12 \mathrm{~Hz}, 14 \mathrm{~Hz}, 16$ $\mathrm{Hz}$ and $18 \mathrm{~Hz}$.

EEG recognition module: The main function of this part is to process the collected SSVEP-EEG, filter EEG by using TMS320F2812 DSP chip, and then perform feature extraction and pattern classification. Convert the classification result into control command and send it to the communication module of Zigbee.

Transmission and control module: This part is mainly to receive the control commands transmitted by the DSP device, and then carry it to the furniture (such as: electric fans, curtains, lamps, etc.), in order to achieve the purpose of controlling the furniture by the brain.

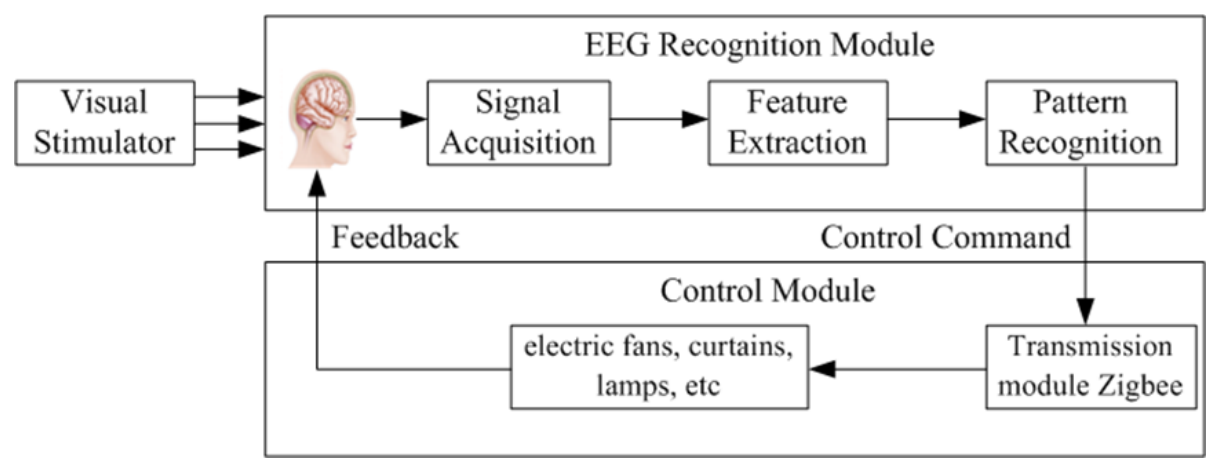

Fig. 1: Block diagram of the system.

\section{Signal Processing}

To realize the control of the external devices by the brain, the identification and classification of EEG are essential. In order to distinguish the EEG under different states which represent different control message. The Fig. 2 displays the processing flow of EEG.

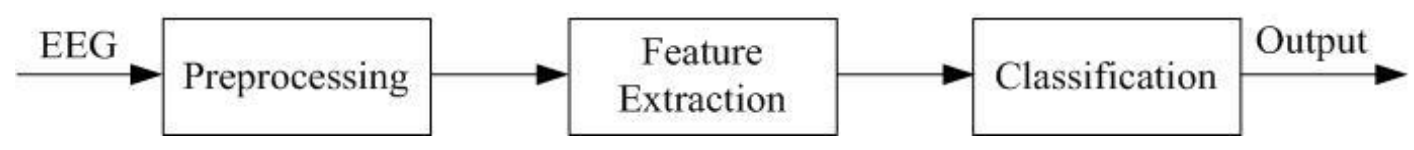

Fig. 2: Signal processing flow chart.

\subsection{Preprocessing}

FIR filters are used in the part.

(1) Filter Structure

The structure of a typical FIR filter ${ }^{[5]}$ is shown in Fig. 3.

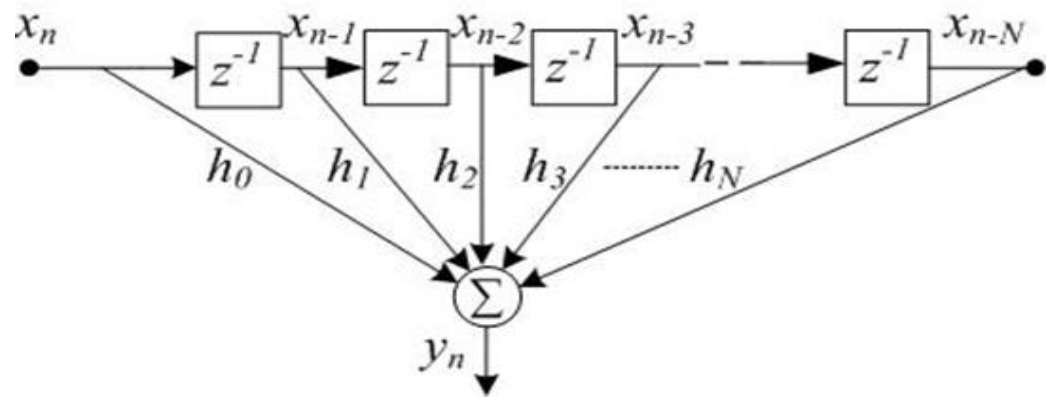

Fig. 3: Structure of FIR filter.

The formula is 


$$
y(n)=\sum_{k=0}^{N} h_{k} x(n-k)
$$

(2) Calculation of Filter Coefficients

The filter coefficients are calculated using the FDATool toolbox in Matlab. After calling FDATool in the command window, select the "target" option in the popup menu, then select "Generate C Header ...", after that select signed 16-bit integer.

(3) Implementation of FIR Filter on DSP

The realization of EEG filtering using FIR in the TMS320F2812 is as follows:

//the calculation of FIR.

for $($ index $=0$; index $<2 * N$;index ++ )

$\{$ input=result $[$ index $]$;

lpf.input=input;// The input function stars to handle the data.

lpf.calc(\&lpf);// lpf function processing

output=lpf.output;

out=(int)output;

yn $[$ index $]=$ out;

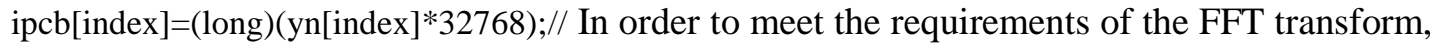

the data needs to be type-converted into the 32-bit long integer. $\}$

In the TMS320F2812, FIR filter is used. EEG before and after filtering are shown in Fig. 4 and Fig. 5, when the stimulation frequency is $12 \mathrm{~Hz}$ and $14 \mathrm{~Hz}$.

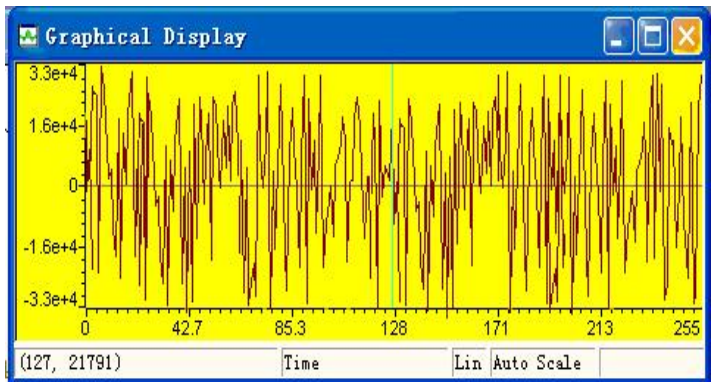

(a)

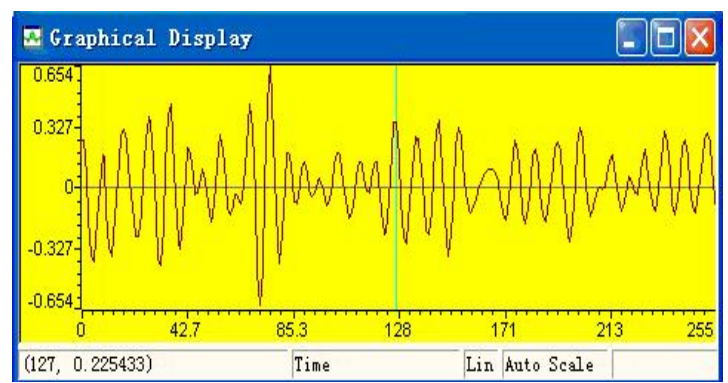

(b)

Fig. 4: Original EEG and the filtered EEG in $12 \mathrm{~Hz}$.

(a) Original EEG; (b) Filtered EEG.

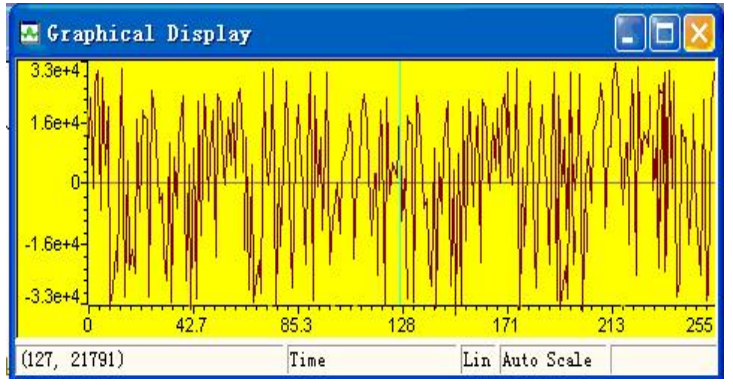

(a)

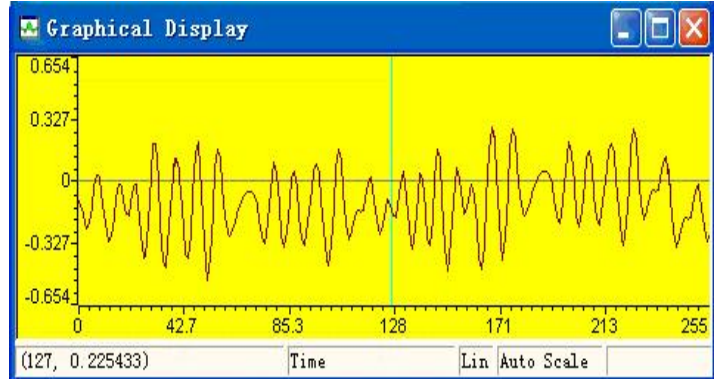

(b)

Fig. 5: Original EEG and the filtered EEG in $14 \mathrm{~Hz}$.

(a) Original EEG; (b) Filtered EEG.

Through the comparison between the filtered EEG and the original EEG, it can be seen that FIR filter can improve the signal to noise ratio well, thereby enhance the collection effect.

\subsection{Feature Extraction}

(1) Fast Fourier Transform

The Fast Fourier Transform (FFT) is a fast algorithm of the discrete Fourier transform(DFT) ${ }^{[6]}$. There are a total of $\mathrm{N}$ points, so it takes $\mathrm{N}^{2}$ complex multiplications and $\mathrm{N}^{*}(\mathrm{~N}-1)$ complex additions to complete the 
entire DFT. Consequently, the computation is quite large. If $W_{N}=e^{-j \frac{2 \pi}{N}}$, the coefficients in the DFT will be $W_{N}^{n k}$, which have the following characteristics:

- $\quad$ Symmetry, $\left(W_{N}^{n k}\right)^{*}=W_{N}^{-n k}$

- Periodicity, $W_{N}^{n k}=W_{N}^{(n+N) k}=W_{N}^{n(k+N)}$

- Reducibility, $W_{N}^{n k}=W_{m N}^{m n k}, W_{N}^{n k}=W_{N / m}^{n k} / m$

Using the features of the Fourier coefficients, some items in DFT can be combined or long sequences can be decomposed into short sequences, thus greatly reducing the amount of calculations of DFT. The FFT is developed based on this idea.

The basic algorithms of FFT include decimation in time (DIT) and decimation in frequency. In this paper, radix-2 DIT-FFT is mainly used.

If the points of the signal $x(n)$ is $\mathrm{N}=2^{l}$, where $l$ is an integer. The signal can be divided into two parts according to the parity of $n$.

$$
x(2 q)=x_{1}(q), x(2 q+1)=x_{2}(q)
$$

So the DFT of $x(n)$ is:

$$
X(k)=\sum_{q=0}^{\frac{N}{2}-1} x_{1}(q)\left(W_{N}^{2}\right)^{q k}+W_{N}^{k} \sum_{q=0}^{\frac{N}{2}-1} x_{2}(q)\left(W_{N}^{2}\right)^{q k}
$$

with the considering of the reducibility of DFT coefficients, there will be

$$
X(k)=\sum_{q=0}^{\frac{N}{2}-1} x_{1}(q) W_{N / 2}^{q k}+W_{N}^{k} \sum_{q=0}^{\frac{N}{2}-1} x_{2}(q) W_{N / 2}^{q k}, k=0,1, \cdots, \frac{N}{2}-1
$$

At this time, the DFT of N/2 points is finished. Utilizing the periodicity of DFT coefficients to compute the DFT of the other N/2 point, there will be,

$$
X\left(k+\frac{N}{2}\right)=\sum_{q=0}^{\frac{N}{2}-1} x_{1}(q) W_{N / 2}^{q k}-W_{N}^{k} \sum_{q=0}^{\frac{N}{2}-1} x_{2}(q) W_{N / 2}^{q k}, k=0,1, \cdots, \frac{N}{2}-1
$$

With this method, DFT of all points can be calculated in the range 0 to $\mathrm{N}-1$ by calculating the DFT of the points from 0 to N/2-1 which involve both odd and even items. The amount of computation is effectively reduced.

(2) Implementation of FFT in DSP

The FFT calculation of the filtered EEG in the TMS320F2812 is as follows:

//Calculate FFT

fft.ipcbptr=ipcb;//The cal function starts to process data.

fft.magptr=mag;//The mag function begin to place squared data.

fft.init(\&fft);// Copy rotation factor FFT

RFFT32_brev(ipcb,ipcb,2*N);// Reverse processing

fft.calc(\&fft);// Call N-point complex FFT calculation, the input data is Q31 format

fft.split(\&fft);// Normalized processing (splitting the N-point complex numbers to get the true result of the $2 \mathrm{~N}$ point $\mathrm{FFT}$ calculation)

fft.mag(\&fft);// The sum of squares of the imaginary part of the real part (the square of the amplitude is obtained and the output data is Q30 format)

for(index $=0$; index $<257$; index ++ )

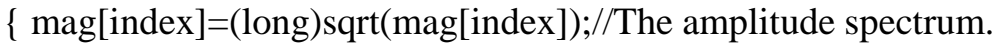

$\operatorname{mag}[0]=0 ;\}$ 
In the TMS320F2812, the spectra of EEG at the stimulation frequency of $12 \mathrm{~Hz}$ and $14 \mathrm{~Hz}$ are shown in Fig. 6 and Fig.7.

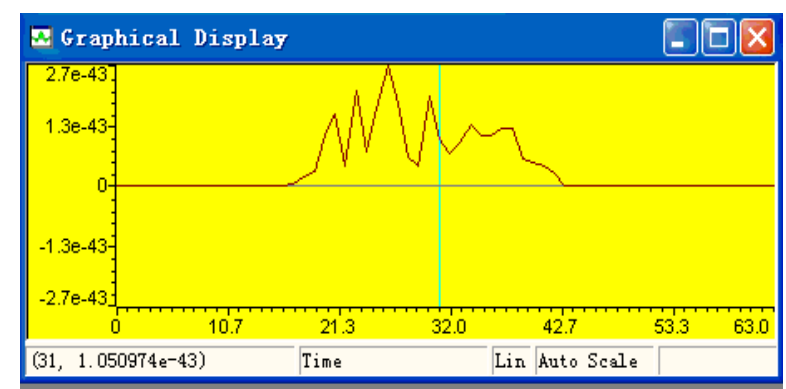

Fig. 6: Spectra of EEG at the stimulation frequency of $12 \mathrm{~Hz}$.

As can be seen from Fig. 6, around the 24th point, the amplitude of the corresponding signal in EEG is significantly increased, whereas there is no obvious change in other signals. So the signal of $12 \mathrm{~Hz}$ can be effectively collected after classification.

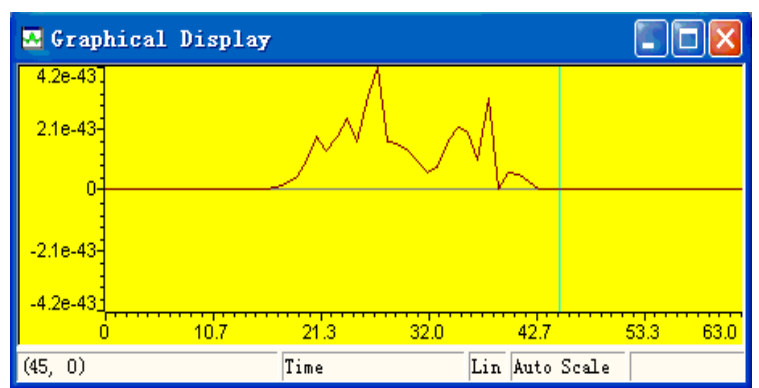

Fig. 7: Spectra of EEG at the stimulation frequency of $14 \mathrm{~Hz}$.

Similarly, the signal of $14 \mathrm{~Hz}$ can be finally selected after classification, which is a good effect.

\section{Wireless Communication Module of Zigbee}

The system establishes a wireless transceiver device consisting of a Zigbee wireless module and a relay module. After the Zigbee wireless module receives a control command through the serial port, the wireless module transmits the command to the slave module of Zigbee which is connected to the furniture, and the relay can control the switch for the furniture. The state of the relay, once the wireless module the slave receives the host's instruction, is judged immediately, thereby completing the intelligent control of the brain on the designated furniture [7-8].

\section{Experiment of the BCI System Based on DSP and Zigbee}

The experiment of the BCI system based on DSP and Zigbee is executed to realize the control of the household appliances. The controlled objects are the electric fan, the desk lamp, the curtain and the door which regard the corresponding EEG at the frequency of $12 \mathrm{~Hz}, 14 \mathrm{~Hz}, 16 \mathrm{~Hz}$ and $18 \mathrm{~Hz}$ signals as the switch.

\subsection{Experiment}

The subjects are 5 healthy students aged from 23 to 26 in the laboratory, of whom 2 are males and 3 are females. The experiment preparations are set as follows:

(1) The subjects sit comfortably in front of the visual stimulator with a distance of $50 \mathrm{~cm}$. The subjects are required to stare at the stimulators horizontally.

(2) Place the electrode. The silver disk electrode should be placed at $\mathrm{O} 2$ of the occipital region of the subject's scalp, which is the recorded channel. To ensure that the EEG can be accurately recorded, the impedance between the electrode and the scalp must be reduced, so the degreasing of the scalp, especially at the place where the electrode is placed, is of the essence with a medical alcohol with the concentration of $75 \%$. The reference electrodes are set in both ears. 
(3) The subjects are informed with the whole process of BCI.

(4) Set parameters. Set the frequency of the stimulator and select the acquisition channel. The sampling frequency is set to $100 \mathrm{~Hz}$.

(5) After the experiment begins, the experimental environment is kept quiet and the subjects focused on the experiment.

The test is carried out as follows:

(1) The subject is instructed to open or close any of the four household appliances.

(2) Run the control system program. According to the appliance provided, the subject selects and stares at the corresponding stimulator to turn on or off the corresponding appliance.

\subsection{Results and Analysis}

The experiment is performed in 5 subjects. There will be 30 trails for each subject. The total consuming time and the accuracy of command are recorded. Tab. 1 shows the statistical results of all subjects.

Table 1: Statistical results of all subjects

\begin{tabular}{ccccc}
\hline Subjects & Consuming time/s & Correct number/times & accuracy/\% & Mean time/(s/time) \\
\hline SY & 150 & $26 / 30$ & $86.7 \%$ & 5 \\
\hline GX & 145 & $26 / 30$ & $86.7 \%$ & 4.8 \\
\hline HY & 140 & $27 / 30$ & $90 \%$ & 4.7 \\
\hline GXH & 150 & $27 / 30$ & $90 \%$ & 5 \\
\hline LYX & 140 & $26 / 30$ & $86.7 \%$ & 4.7 \\
\hline
\end{tabular}

It can be verified that the recognition accuracy of the designed BCI system based on DSP and Zibee can reach more than $85 \%$. Furthermore, the consuming time to turn on or off any household appliance is less than $5 \mathrm{~s}$. It is proved that the proposed BCI system based on DSP and Zibee is feasible and has high application value.

\section{Conclusion}

BCI, as a research, is not only a hotspot in cross-discipline of life sciences and information technology, but also a difficulty, which provides a way for people with normal thinking and movement disorders to communicate with others and control some facilities in the medical field, at the same time, establish a special access to communicate or control in particular environment such as the aerospace. However, because EEG is non-stationary, strongly nonlinear and random, its regularity has not yet been fully recognized. There has been no breakthrough in the analysis and processing of EEG, which caused only a few results have been able to be applied in practice. It is strongly believed that with the steady development, it can play an important role in the disability engineering, aerospace engineering, military fields and so on. It will have much higher technologies that can be applied to more fields on account of its practicality. Ultimately, BCI must benefit humanity.

\section{Acknowledgement}

The paper was supported by the Scientific Research Development Fund Project of Tianjin University of Technology and Education (KJ1804).

\section{References}

[1] Zhonglin Lin, Changshui Zhang, Wei Wu, Xiaorong Gao. Frequency Recognition Based on Canonical Correlation Analysis for SSVEP-based BCIs. IEEE Transactions on Biomedical Engineering. 2006, 53 (12): 2610-2614.

[2] Li Zhao, Yong Sun, Xuhong Guo, et al. Research on Mobile Phone Dialing System Based on Steady-State Visual Evoked Potential. Chinese Journal of Biomedical Engineering. 2013, 32 (2): 253-256.

[3] Guangyu Bin, Xiaorong Gao, Zheng Yan, Bo Hong, Shangkai Gao. An Online Multi-channel SSVEP-based Braincomputer Interface Using a Canonical Correlation Analysis Method. Journal of Neural Engineering. 2009, 6 (4): 16 
[4] Guangyu Bin, Zhonglin Lin, Xiaorong Gao, et al. The SSVEP Topographic Scalp Maps by Canonical Correlation Analysis. Proceedings of the 30th Annual International Conference of the IEEE Engineering in Medicine and Biology Society. 2008, pp. 3759-3762.

[5] M. Stepien, J. Conradi, G. Waterstrat, et al. Event-related Desynchronization of Sensorimotor EEG Rhythms in Hemiparetic Patients with Acute Stroke. Neuroscience Letters. 2011, 488 (1): 17-21.

[6] Li Zhao, Yong Sun, Yang He, Zhenyan Ma. Research of Brain-computer Interface Automatic Vehicle System Based on SSVEP. Electronic Measurement Technology. 2011, 34 (12): 70-72.

[7] J.R. Wolpaw, N. Birbaumer, W.J. Heetderks. Brain-computer Interface Technology: A Review of the First International Meeting. IEEE Transactions on Rehabilitation Engineering. 2000, 8 (2): 164-173.

[8] Feng Xu, Xin Liu, Jiabao Fang. Design of Smart Home Remote Control System. Electrical \& Energy Management Technology. 2009, (4): 21-24. 